\section{The American College of Rheumatology/European League Against Rheumatism Criteria for the classification of rheumatoid arthritis: a game changer}

\author{
Stanley Cohen,1 Paul Emery²
}

Over the last several years the two preeminent professional societies representing rheumatology, the American College of Rheumatology (ACR) and the European League Against Rheumatism (EULAR), have been discussing increasing their collaboration in areas of interest to rheumatologists worldwide. These discussions resulted in a letter of agreement in 2008 on the framework whereby the two organisations would work together to develop disease classification criteria as well as recommendations for conducting of clinical trials. To enhance communication between the two organisations, ACR representatives now sit on the EULAR Standing Committee for Clinical Affairs and the EULAR Standing Committee of Epidemiology, and EULAR representatives now sit on the ACR Criteria Subcommittee and Quality of Care Committee.

The first result of this effort was the joint publication, in Annals of the Rheumatic Diseases and Arthritis Care \& Research, of the recommendations on reporting disease activity in clinical trials of patients with rheumatoid arthritis (RA). ${ }^{1} 2$ This document was important in that it delineated the minimal standards necessary for clinical trials evaluating new therapeutics in RA. Several collaborative projects are under way on RA, and also on gout, scleroderma, myositis and vasculitis. The positive aspects of developing a consensus between the dominant voices in world of rheumatology are selfevident.

\footnotetext{
${ }^{1}$ Metroplex Clinical Research Center, University of Texas Southwestern Medical School, Dallas, Texas, USA; ${ }^{2}$ University of Leeds and NIHR Leeds Musculoskeletal Biomedical Research Unit, Leeds, UK
}

Correspondence to Dr Stanley Cohen, 5939 Harry Hines Boulevard, Suite 400, Dallas, TX 75229, USA; arthdoc@aol.com

This article is published simultaneously in the September 2010 issue of Arthritis \& Rheumatism
The recent initiative to revise the ACR classification criteria for $\mathrm{RA}^{3}$ is the most significant cooperation so far. For the past 2 years rheumatologists on both sides of the Atlantic have been working on this project, and the fruits of their work are now published in this journal.

The last classification criteria were published in $1987,{ }^{5}$ and are widely regarded as unsatisfactory for the diagnosis of RA (for which they were not designed). The need for the new classification criteria has been made more urgent by the understanding that, at presentation, RA may be an evolving disease, the final phenotype of which can be altered by interventions. From work in European clinics evaluating patients presenting with early undifferentiated arthritis, it was clear that the discriminant ability of the previous RA classification criteria was insufficient to distinguish those patients destined eventually to develop RA from those who would have a limited course or whose condition would evolve into other forms of inflammatory arthritis. ${ }^{6}$ Additionally, the developing science regarding the importance of antibodies to citrullinated proteins in $\mathrm{RA}^{7}$ occurred subsequent to the last classification criteria, and it was clear that inclusion of this testing in updated criteria was critical. Over the last two decades, early intervention to prevent functional decline has become accepted as the standard of care. The ideal therapeutic intervention would be undertaken at an early stage before the development of the final phenotype, described by the previous classification criteria, producing a positive impact on disease progression in the majority of patients.

The joint working group, realising the deficiencies of the previous criteria, set out with several major goals. These included identifying, among patients with newly presenting undifferentiated arthritis, a subset with a high risk of chronicity and erosive damage, and ensuring that the new criteria could be used as a basis for initiating diseasemodifying antirheumatic drug therapy. A comprehensive programme was developed and conducted in three phases. In Phase 1, utilising a data-driven approach based on three cohorts of patients with early arthritis, the working group identified factors that were associated with the subsequent decision by physicians to initiate methotrexate therapy, and their relative weights. Phase 2 was consensus driven, with a science-based approach informed by data from Phase 1. Phase 3 was the derivation from the previous two phases: the final classification criteria set. The final criteria set was validated with three cohorts not used in Phase 1. The criteria are thus a mix of pragmatic expert opinion and a sciencedriven approach.

As noted by the working group, utilisation of these criteria 'redefines the current paradigm of RA'. The 1987 classification criteria were useful in discriminating patients with RA from those with other inflammatory arthritides, but have not been helpful in identifying patients who would potentially benefit from early intervention. The new criteria differ from the previous criteria in that the presence of synovitis in at least one joint is required, with no alternative diagnosis to explain the synovitis. Symmetric disease involvement is not required, nor is the presence of structural joint damage or rheumatoid nodules, both reflective of longstanding, established disease. A scoring system evaluating four categoriesjoint involvement, serology, acute-phase response and duration of symptomshas been developed. Using this new scoring system, 87-97\% of patients in three early arthritis cohorts in whom methotrexate treatment was begun within 12 months from symptom onset met the new classification criteria for 'definite RA'. Importantly, the domains assessed have face validity, as was predicted several years ago. ${ }^{8}$ The new criteria provide a more rigorous scientific basis for this approach.

We believe these new classification criteria will be rapidly adopted in daily practice, and we look forward to their implementation in clinical trials. Certainly this will accelerate the use of more aggressive treatment for patients and, as the authors note, additional studies in different clinical settings need to be conducted to determine their applicability. How these criteria might impact 
patient selection for clinical trials will be of great interest.

Change can be difficult for a generation of rheumatologists used to classifying RA with the old criteria. Concerns over the absence of erosion in the scoring system, as well as the absence of the necessity of symmetric joint involvement, will be raised. The working group does acknowledge that the presence of erosions typical of RA would justify classification of a patient as having RA, but also raises the question of what is meant by significant erosive disease and what evidence of erosions should be considered acceptable as signifying 'typical of RA'. Symmetric joint disease was not found to provide additional independent weight to the criteria. Additional concerns exist regarding the utility of these classification criteria for the primary care physician who must determine synovitis by examination and then exclude other possible diagnoses that might explain the synovitis. The authors correctly point out that the criteria are not to be used as a tool for referral of patients with inflammatory arthritis to the rheumatologist, and there are several ongoing efforts in progress to provide primary care practitioners with the tools to recognise patients who need rapid, early referral.

It might be predicted that classic pharmaceutical studies of 'early' active RA will be unchanged, since the vast majority of these patients with a high disease activity score and frequent radiological erosions have an advanced phenotype. The exciting new area will be patients previously labelled as having undifferentiated arthritis, with one to two swollen joints and anticitrullinated protein antibody positivity, who may well score sufficiently to be labelled as having RA. This should encourage studies of the disease at this crucial stage of evolution. For these patients, the issue of defining synovitis and, as noted above, a 'typical' erosion will need to be evaluated, and the current subjective clinical diagnosis may need refinement using objective and more sensitive imaging modalities, such as MRI and ultrasound.

We applaud the efforts of all involved in the development of the new RA classification criteria. Prior to publication, the manuscript was critically reviewed not only by the journal editors and reviewers, but also by leadership of both the ACR and EULAR, including the boards of directors and committee members. That input was important in the eventual publication of this straightforward and well-written document. ${ }^{3}$ The acceptance of the evolving nature of RA is a stepchange conceptually. We look forward to the identification of future biomarkers that will again result in another call to modify the RA classification criteria. When that occurs, improvement in the quality of life of our patients will surely follow.

\section{Competing interests None.}

Provenance and peer review Not commissioned; not externally peer reviewed.

Contributors Drs Cohen and Emery drafted and revised the article and approved the final version to be published.
Accecpted 28 June 2010

Ann Rheum Dis 2010;69:1575-1576

doi:10.1136/ard.2010.138446

\section{REFERENCES}

1. Aletaha D, Landewe R, Karonitsch T, et al. Reporting disease activity in clinical trials of patients with rheumatoid arthritis: EULAR/ACR collaborative recommendations. Ann Rheum Dis 2008;67:1360-4

2. Aletaha D, Landewe R, Karonitsch T, et al. Reporting disease activity in clinical trials of patients with rheumatoid arthritis: EULAR/ACR collaborative recommendations. Arthritis Rheum 2008;59:1371-7.

3. Aletha D, Neogi T, Silman AJ, et al. 2010 Rheumatoid arthritis classification criteria: an American College of Rheumatology/ European League Against Rheumatism collaborative initiative. Arthritis Rheum 2010;62:2569-81.

4. Aletha D, Neogi T, Silman AJ, et al. 2010 Rhuematoid arthritis classification criteria: an American College of Rheumatology/European League Against Rheumatism collaborative initiative. Ann Rheum Dis 2010;69:1580-88

5. Arnett FC, Edworthy SM, Bloch DA, et al. The American Rheumatism Association 1987 revised criteria for the classification of rheumatoid arthritis. Arthritis Rheum 1988;31:315-24.

6. Van der Helm-van Mil AH, Detert J, le Cessie $S$, et a/ Validation of a prediction rule for disease outcome in patients with recent-onset undifferentiated arthritis: moving toward individualized treatment decision-making. Arthritis Rheum 2008;58:2241-7.

7. Aggarwal R, Liao K, Nair R, et al. Anti-citrullinated peptide antibody assays and their role in the diagnosis of rheumatoid arthritis. Arthritis Rheum 2009;61: 1472-83.

8. Emery P. The Dunlop-Dottridge Lecture: prognosis in inflammatory arthritis: the value of HLA genotyping and the oncological analogy. J Rheumato/1997;24 $1436-42$ 
Corrections

doi:10.1136/ard.2010.138446corr1

Cohen S, Emery P. The American College of Rheumatology/European League Against Rheumatism Criteria for the classification of rheumatoid arthritis: a game changer. Ann Rheum Dis

2010;69:1575-76. On page 1575 'based on three cohorts' should read 'based on six cohorts.' 